\title{
Assessment, prevention and treatment of osteoporosis in inflammatory bowel disease patients: a bone of contention
}

\section{Stephen B Hanauer}

In this issue of Nature Clinical Practice Gastroenterology \& Hepatology Bernstein and Leslie review the evidence regarding the risk of osteoporosis and fractures in patients with inflammatory bowel disease (IBD). The authors base their review and discussion on the discrepancy between observed rates of osteoporosis and fractures in patients with IBD compared with other high-risk populations (postmenopausal women and those at risk for corticosteroid-induced osteoporosis). The authors offer very rational advice regarding treatment approaches for the underlying disease, but are quite skeptical regarding the role of bisphosphonates for preventing fractures, despite their impact on bone density. Their skepticism also extends to the utility of bone mineral density (BMD) testing in the IBD population and they urge that there be more 'selectivity' in deciding when it is performed. This Review provides an opportunity to discuss some of the controversies as well as potential approaches to overcoming the bipartisan debate surrounding the risk, prevention and management of osteoporosis and fractures in IBD patients.

The primary reason for the controversy surrounding this issue is that most data related to osteoporosis derive from either the postmenopausal-female population or from patients exposed to corticosteroids (most often, women with rheumatologic disorders). Both of these groups typically comprise a much older population, and there is a predominance of women and patients who are less mobile because of age or arthritis. Furthermore, as the dual-energy X-ray absorptiometry scans used for BMD testing are interpreted according to T-scores, the reduced bone densities observed in younger individuals are still greater than reduced bone densities seen in older individuals. It is therefore not unexpected that fracture rates are going to be lower in patients with IBD, despite their 'low' bone density. The ultimate question that needs to be addressed is whether continuing low bone density in the young IBD population will lead to an increased risk of fractures as these patients age.
In the absence

of definitive

evidence...

I favor

prevention and

treatment of

osteoporosis

in IBD patients

in the attempt

to prevent

osteoporotic

fractures as

these patients

age

SB Hanauer is Editorin-Chief of Nature Clinical Practice Gastroenterology \& Hepatology.

Competing interests

The author declared competing interests; go to the article online for details.

www.nature.com/clinicalpractice doi:10.1038/ncpgasthep0176
Potential risk factors for reduced bone density in IBD patients include underlying inflammatory disease, involvement of the ileum (where fat-soluble vitamins are absorbed), malnutrition (more often seen in Crohn's disease), small stature, smoking (more common in Crohn's disease) and use of glucocorticoids. The overall risk of osteoporosis is higher in patients with Crohn's disease than with ulcerative colitis owing to concurrent risk factors of smoking, malnutrition, ileal disease and perhaps (until recently), glucocorticoid dependence. Another important risk factor is family history (presumably genetic). All clinicians have observed patients at risk of developing osteoporosis because of all of the aforementioned factors, yet who have no evidence of bone demineralization, despite prolonged corticosteroid treatment.

The difficulty in interpreting data regarding bisphosphonates relates to the heterogeneity of the IBD population, concomitant risk factors, and the lack of long-term data. Meanwhile, the potential value of supplementation with calcium and vitamin D far outweighs any risk. In addition to their potential impact on bone, these supplements can reduce the risk of nephrolithiasis and might have chemopreventive benefits with regard to inflammation and carcinogenesis. What are greatly needed are trials to determine whether bisphosphonates can prevent the rapid bone loss associated with glucocorticoid therapy in this population (a 3-month trial would suffice) and data comparing the bone-density scores of age-matched patients over 50 with osteoporosis with those of other at-risk populations. In fact, elderly osteoporotic IBD patients will develop fractures at higher rates to non-IBD individuals.

Meanwhile, I agree that 'selectivity' should determine which IBD patients are evaluated for metabolic bone disease. In the absence of definitive evidence, however, I favor prevention and treatment of osteoporosis in IBD patients in the attempt to prevent osteoporotic fractures as these patients age. 